\title{
Transition phenomenon in $\mathrm{Ti}_{2} \mathrm{O}_{3}$ using the discrete variational $X \alpha$ cluster method and periodic shell model
}

\author{
H. Nakatsugawa and E. Iguchi* \\ Materials Science, Department of Mechanical Engineering and Materials Science, Faculty of Engineering, \\ Yokohama National University, Tokiwadai, Hodogaya-Ku, Yokohama, 240 Japan
}

(Received 17 March 1997; revised manuscript received 14 July 1997)

\begin{abstract}
The electronic structure of the metallic $\mathrm{Ti}_{2} \mathrm{O}_{3}$ and insulating phases has been investigated using a combination of the three-dimensional periodic shell model and the discrete-variational (DV)- $X \alpha$ cluster method. Besides the effects of intersite repulsive nearest-neighbor electron-electron $(d-d)$ Coulombic interaction and the spin-spin interaction by means of a generalized Hubbard Hamiltonian, the Hamiltonian in the insulating phase includes Anderson's attractive potential due to the electron-phonon interaction. The shell model estimates the electron-phonon coupling constants, and provides direct theoretical evidence that the electronphonon interaction stabilizes the three dimensional periodic distribution of $\mathrm{Ti}^{3+}-\mathrm{Ti}^{3+}$ pairs along the $c$ axis of the corundum structure. The DV- $X \alpha$ cluster method calculates the electron energies in the $\left[\mathrm{Ti}_{2} \mathrm{O}_{9}\right]^{-12}$ cluster, the values for the intersite repulsive nearest-neighbor $d$ - $d$ interaction, and the spin-spin interaction. The electron correlation effect and the spin correlation effect are found to be mainly responsible for the broad crossover between a metallic and an insulating state in $\mathrm{Ti}_{2} \mathrm{O}_{3}$, although there is some contribution from the electron-phonon interaction. The present calculation describes the characteristic transition phenomenon in this material. [S0163-1829(97)07744-8]
\end{abstract}

\section{INTRODUCTION}

Unlike the clear cut metal-insulator (MI) transition in $\mathrm{VO}_{2}$, titanium sesquioxide $\mathrm{Ti}_{2} \mathrm{O}_{3}$ exhibits a broad crossover between a metallic and an insulating state around $T_{c}$ $\cong 450 \mathrm{~K},{ }^{1}$ which is also an MI transition. Below $T_{c}, \mathrm{Ti}_{2} \mathrm{O}_{3}$ indicates the presence of a finite energy gap $E_{G}$ between the occupied $a_{1 g}$ band and the unoccupied $e_{g}^{\pi}$ band. In fact, specific-heat studies, conductivity measurements, thermoelectric measurements, and other experiments yield $E_{G}$ $\simeq 0.1 \mathrm{eV}$ at $300 \mathrm{~K}^{2-6}$ These experimental results require calculations of electronic structures in insulating and metallic phases, because the transition of the insulating state to the metal should be supported theoretically by band structures. Despite extensive experiments over the various properties of $\mathrm{Ti}_{2} \mathrm{O}_{3},{ }^{7-18}$ however, details of the band structures are still unknown. Goodenough and co-workers tried an explanation for this transition using a simple crystal-field theory based upon the Mott-Hubbard regime. ${ }^{19-21}$ It is generally accepted that a broad crossover between $a_{1 g}$ and $e_{g}^{\pi}$ bands at different points in the Brillouin zone with increasing temperature results in the transition of $\mathrm{Ti}_{2} \mathrm{O}_{3}$, with the resulting disappearance of the energy gap at $T_{c} \cdot{ }^{19,20}$

Conventional band calculations created no energy gap, ${ }^{22,23}$ although their numerical estimate for the $a_{1 g}$ bandwidth was nearly equal to the experimental value obtained by angle-resolved ultraviolet-photoemission spectroscopy (ARUPS). ${ }^{17,18}$ According to Zeiger and co-workers, ${ }^{24,25}$ the main driving force for the transition phenomenon in $\mathrm{Ti}_{2} \mathrm{O}_{3}$ is competition between the electron-electron correlation energy and the lattice-displacement energy due to formation of singlet excitons. The theoretical construction of the band structure in $\mathrm{Ti}_{2} \mathrm{O}_{3}$ has to take account of electron correlation and spin correlation effects which were not involved in the pre- vious theoretical trials. ${ }^{19-25}$ Although the electron-phonon interaction effect looks not so strong in $\mathrm{Ti}_{2} \mathrm{O}_{3}$ compared with $\mathrm{VO}_{2},{ }^{22}$ the low-frequency $A_{1 g}$ vibration mode in $\mathrm{Ti}_{2} \mathrm{O}_{3}$ requires that this effect is also to be included in the band calculation.

Castellani, Feinberg, and Ranninger ${ }^{26}$ developed a Greenfunction formalism which includes the electron correlation effect, the electron-phonon interaction effect, and the spin correlation effect acting on the diamagnetic units of two adjacent $3 d$ electrons. Using this technique, they tried to account for the broad crossover between the metallic and insulating phases in $\mathrm{Ti}_{2} \mathrm{O}_{3}$. Their result indicates that weak electron-phonon interaction induces the decay of the spin correlation effect, which eventually results in the closing of the gap between $a_{1 g}$ and $e_{g}^{\pi}$ bands. In their theoretical treatment, the electron-phonon interaction effect was evaluated by the relative relationship between the intrapair hopping integral, in the $c$ direction, and the interpair hopping integral, in the basal plane.

In our previous study ${ }^{27}$ of MI transition effects in $\mathrm{VO}_{2}$, the $d-d$ electron correlation effect and the strong electronphonon interaction effect operating on the $\mathrm{V}^{4+}-\mathrm{V}^{4+}$ pairs were assessed by using the combination of the threedimensional periodic shell model and the discrete-variational (DV) $-X \alpha$ cluster method. Particularly, the shell model estimated the electron-phonon interaction energies independently. Consequently, the quantitative analysis for the MI transition in $\mathrm{VO}_{2}$ has progressed remarkably. In the present report, then, we applied this treatment to the transition phenomenon in $\mathrm{Ti}_{2} \mathrm{O}_{3}$.

As shown in Fig. $1, \mathrm{Ti}_{2} \mathrm{O}_{3}$ has a corundum $\left(\alpha-\mathrm{Al}_{2} \mathrm{O}_{3}\right)$ structure with a trigonal Bravais lattice (rhombohedral, space group $R \overline{3 c}$ ) at all temperatures, and consists of a hexagonalclose-packed oxygen lattice where $\mathrm{Ti}^{3+}$ ions fill two-thirds of 


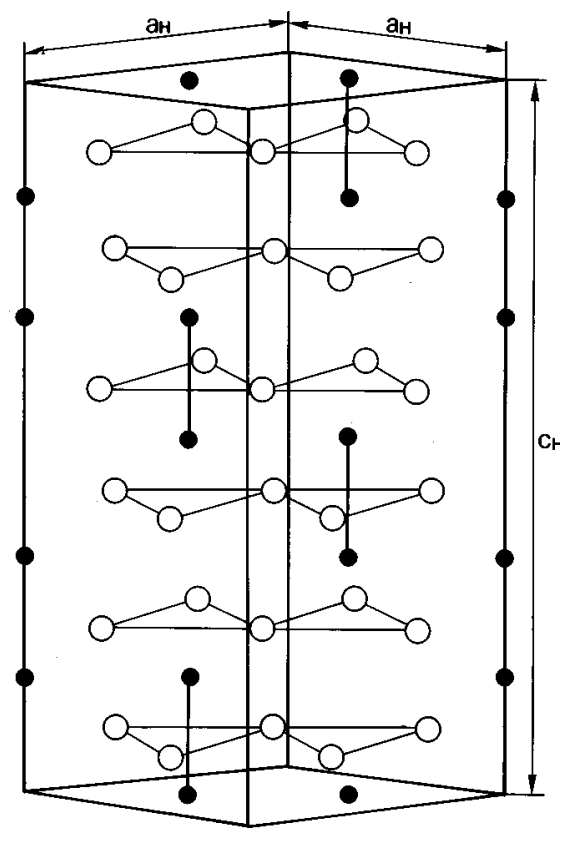

FIG. 1. The corundum structure of $\mathrm{Ti}_{2} \mathrm{O}_{3}$ with the lattice constants parallel and perpendicular to the $c$ axis, $c_{H}$ and $a_{H}$. Closed circles are $\mathrm{Ti}^{3+}$ ions, and open ones $\mathrm{O}^{2-}$.

the octahedral interstices. ${ }^{28-31}$ The hexagonal unit cell consists of a 30-atom basis set made up of six " $\mathrm{Ti}_{2} \mathrm{O}_{3}$ " molecular units. Two $\mathrm{Ti}^{3+}$ ions in a " $\mathrm{Ti}_{2} \mathrm{O}_{3}$ " molecule are located at special positions along the threefold axis at $\left[0,0, z ; 0,0, \frac{1}{2}\right.$ $+z]$ and three $\mathrm{O}^{2-}$ ions are located at $\left[x, 0, \frac{1}{4} ; 0, x, \frac{1}{4} ; \bar{x}, \bar{x}, \frac{1}{4}\right] .{ }^{30}$ Then $z$ and $x$ are the atomic parameters which depend upon temperature. In the insulating phases at 300 and $390 \mathrm{~K}$, $(z, x)=(0.3445,0.3133)$ and $(0.3450,0.3133)$. Then $\mathrm{Ti}_{2} \mathrm{O}_{3}$ contains the three-dimensional periodic distribution of $\mathrm{Ti}^{3+}-\mathrm{Ti}^{3+}$ couples paired along the $c$ direction. There is another $\mathrm{Ti}^{3+}-\mathrm{Ti}^{3+}$ couple in the basal plane normal to the $c$ axis. The ionic spacing of $\mathrm{Ti}^{3+}-\mathrm{Ti}^{3+}$ couples parallel to the $c$ axis is short compared with that in the basal plane. As temperature lowers, the short $\mathrm{Ti}^{3+}-\mathrm{Ti}^{3+}$ separation along the $c$ axis decreases, while the long one in the basal plane increases. ${ }^{28-31}$ Thus a strong interaction working on the pairs along the $c$ axis leads to direct bond formation between the cations, and plays a crucial role in the transition. ${ }^{19}$

$\mathrm{Ti}^{3+}-\mathrm{Ti}^{3+}$ separations are very sensitive to the stability of the $a_{1 g}$ and $e_{g}^{\pi}$ bands. ${ }^{20}$ When temperature rises across $T_{c}$, the $c_{H} / a_{H}$ ratio increases, ${ }^{19-21}$ where $c_{H}$ and $a_{H}$ are the lattice constants parallel and perpendicular to the $c$ axis. This implies that the partial occupation of the $e_{g}^{\pi}$ states with increasing temperature enhances the increase in the $c_{H} / a_{H}$ ratio, reducing the covalency of $\mathrm{Ti}^{3+}-\mathrm{Ti}^{3+}$ pairs. Thus there is a correlation between the transition and the covalency of the $\mathrm{Ti}^{3+}-\mathrm{Ti}^{3+}$ bonds along the $c$ axis. However, there is no change in the crystallographic symmetry even if the insulating phase transfers to the metallic phase. ${ }^{19-21}$ This must be mainly because of the low-frequency $A_{1 g}$ vibration mode which does not lower the lattice symmetry. At $300 \mathrm{~K}, \mathrm{Ti}_{2} \mathrm{O}_{3}$ involves only this vibration mode. ${ }^{21}$ Then the electronphonon interaction effect induced by this mode is expected to play an important role in the stability of the insulating phase.

As described in our previous paper, ${ }^{27}$ the eigenvalue of
Anderson's Hamiltonian, ${ }^{32}$ including his attractive potential ${ }^{33}$ in an insulating phase of strongly correlated oxides like $\mathrm{Ti}_{2} \mathrm{O}_{3}$, is expressed as ${ }^{33-35}$

$$
E=2 \varepsilon_{i}+U-\frac{\lambda^{2}}{2 c},
$$

where $\varepsilon_{i}$ is an atomic energy at the $i$ th site, $U$ represents an intersite nearest-neighbor $d-d$ interaction, $c$ is a relevant elastic constant, and $\lambda$ is an electron-phonon coupling constant between $\mathrm{Ti}^{3+}$ ions on the bond sites. In Eq. (1), " $U$ ", comes from the $d$ - $d$ electron correlation effect and the spin correlation effect, and " $\lambda{ }^{2} / 2 c$ " from the electron-phonon interaction effect. ${ }^{27}$ Thus the occupied $a_{1 g}$ band in the insulating phase lowers in energy by $\lambda^{2} / 2 c$. This is consistent with the idea of Goodenough and co-workers that the energy gap between the occupied $a_{1 g}$ band and the unoccupied $e_{g}^{\pi}$ band increases by the $\mathrm{Ti}^{3+}-\mathrm{Ti}^{3+}$ pairing in the $c$ axis direction. ${ }^{19-21}$ The numerical estimate for $\lambda^{2} / 2 c$ in Eq. (1) requires the calculation of the change in the lattice energy induced by the pairing.

The very slight lattice distortion in the three-dimensional distribution of the $\mathrm{Ti}^{3+}-\mathrm{Ti}^{3+}$ pairs along the $c$ axis imposes stringent constraints on the required accuracy in making the theoretical estimates of relevant parameters. Our threedimensional periodic shell model, ${ }^{36}$ developed from the shell model originated by Dienes et al. ${ }^{37}$ surely meets such a strict requirement, as shown in our study on $\mathrm{VO}_{2}{ }^{27}$ Since the DV- $X \alpha$ cluster method can calculate the energies of electron states ${ }^{38-44}$ the combination of the periodic shell model and the cluster method must contain the possibility to clarify the origin of the characteristic broad crossover between the metallic and the insulating state in $\mathrm{Ti}_{2} \mathrm{O}_{3}$ from the energetic point of view. Therefore, a calculation using this combination is of great significance in order to understand properly the transition phenomenon in $\mathrm{Ti}_{2} \mathrm{O}_{3}$.

\section{THEORETICAL PROCEDURE}

\section{A. Crystal structure of $\mathrm{Ti}_{2} \mathrm{O}_{3}$}

$\mathrm{Ti}_{2} \mathrm{O}_{3}$ has a corundum structure, as described in Sec. I. Changes in the lattice parameters with increasing temperature are nonlinear. ${ }^{28,29} \mathrm{The}^{\mathrm{Ti}}{ }^{3+}-\mathrm{Ti}^{3+}$ separation parallel to the $c$ axis increases smoothly from $2.5818 \AA$ at $300 \mathrm{~K}$ to $2.7227 \AA$ at $868 \mathrm{~K}$, a $5.5 \%$ increase, as the lattice constant $c_{H}$ increases from 13.610 to $13.957 \AA$. On the other hand, the $\mathrm{Ti}^{3+}-\mathrm{Ti}^{3+}$ separation in the basal plane decreases over the same temperature interval from 2.9939 to $2.9854 \AA$, a $0.3 \%$ decrease, while the unit cell parameter $a_{H}$ decreases from 5.1570 to $5.1251 \AA \AA^{28-31}$ Therefore, the $c_{H} / a_{H}$ ratio increases from 2.6391 to 2.7233 , a $3.2 \%$ increase. These changes are consistent with a band-crossing model proposed by Goodenough and co-workers ${ }^{19-21}$ for the transition behavior in $\mathrm{Ti}_{2} \mathrm{O}_{3}$. The present calculation as a parametric function of temperature is based upon these experimental lattice parameters.

The low point symmetry in $\mathrm{Ti}_{2} \mathrm{O}_{3}$ induces a monopole field on every ion. The relaxation due to both the monopole field acting on each ion and the electron-phonon interactions acting on $\mathrm{Ti}^{3+}-\mathrm{Ti}^{3+}$ pairs along the $c$ axis displace all of the ions, and then $\mathrm{Ti}^{3+}-\mathrm{Ti}^{3+}$ bonds are formed. This implies that 
TABLE I. The parameters of $\mathrm{Ti}_{2} \mathrm{O}_{3}$ employed in the shell model calculations: free-ion polarizabilities $\alpha^{0}$, electronic polarizabilities $\alpha^{e}$, average exciting energies $E_{\mathrm{ex}}$, and shell parameters $Q$.

\begin{tabular}{lcccc}
\hline \hline Ion & $\alpha^{0}\left(\AA^{3}\right)$ & $\alpha^{e}\left(\AA^{3}\right)$ & $E_{\text {ex }}(\mathrm{eV})$ & $Q(e)$ \\
\hline $\mathrm{Ti}^{3+}$ & 0.186 & 0.400 & 105.87 & 7.380 \\
$\mathrm{O}^{2-}$ & 3.880 & 2.405 & 8.00 & 1.890 \\
\hline \hline
\end{tabular}

the initial ionic distribution transfers to the final one. The lattice parameters in the insulating $\mathrm{Ti}_{2} \mathrm{O}_{3}$ phase are the ones in the final structure, which are obtained in x-ray analyses. Then the theoretical treatment requires a hypothetical crystal structure in the initial state, which is to be determined in the following way. The relaxation calculation using the threedimensional periodic point-ion shell model ${ }^{27,36}$ has been iterated by changing the initial ionic positions, until the final positions yield the experimental atomic parameters, i.e., $(z, x)=(0.3449,0.3133)$ and $(0.3450,0.3133)$ at 300 and $390 \mathrm{~K}$, respectively. In the end, the atomic parameters in the initial states are $(z, x)=(0.3450,0.3130)$ and $(0.3451$, $0.3130)$ at 300 and $390 \mathrm{~K}$, respectively. Therefore, the $\mathrm{Ti}^{3+}-\mathrm{Ti}^{3+}$ separations parallel to the $c$ axis in the initial states are 2.586 and $2.593 \AA$ at 300 and $390 \mathrm{~K}$. In the final states, they reduce to 2.581 and $2.590 \AA$.

The calculations on the metallic phase at $450 \mathrm{~K}$ were based upon experiments, i.e., $(z, x)=(0.3455,0.3127)$. Similar calculations were carried out at several temperatures in the metallic phase above $450 \mathrm{~K}$. The atomic displacement was not taken into account in the metallic phase for two reasons: (i) the shell model is applicable only in insulating ionic crystals, ${ }^{27,36,37,45-53}$ and (ii) the displacement on each atom in the metallic phase is remarkably small even if the relaxation is calculated by the shell model.

\section{B. Theoretical methods}

The combination of the three-dimensional periodic shell model and the DV- $X \alpha$ cluster method is employed. Since full details of the periodic shell model were published elsewhere, ${ }^{27,36,45-53}$ only a brief description will be given here. The lattice energy in a rigid and unpolarized crystal is given by the sum of the long-range Madelung energy $\left(E_{M}\right),{ }^{54,55}$ the short-range repulsive energy $\left(E_{R}\right)$, and the van der Waals energy $\left(E_{\mathrm{vdW}}\right)$. The Born-Mayer constants and the van der Waals constants included in the lattice energy calculations were obtained using the wave functions of free ions, electronic polarizabilities $\alpha^{e}$, and average exciting energies $E_{\mathrm{ex}}$, calculated by the theoretical procedures ${ }^{56-62}$ which were employed in our previous reports. $27,36,45-53,63-66$ The values of $\alpha^{e}$ and $E_{\mathrm{ex}}$ are collected in Table I together with free-ion polarizabilities of Pauling, ${ }^{67} \alpha^{0}$, and the shell parameters $Q$ which were determined by the method developed from the theory of Dick and Overhauser ${ }^{68}$ by Shanker and Gupta, ${ }^{69,70}$ using electronic polarizabilities. The BornMayer constants of the ion pairs involving $\mathrm{Ti}^{3+}$ ions require a modification from those involving $\mathrm{Ti}^{4+}$ ions based upon the scaling procedure using Shannon and Prewitt's ionic radii. $^{71}$ (A table of the Born-Mayer and van der Waals constants will be furnished by the authors upon request.)
Even in a rigid and unpolarized $\mathrm{Ti}_{2} \mathrm{O}_{3}$ with a lattice energy of $E_{L}$, a relaxation due to monopole fields acting on all of the ions takes place. The total energy of the crystal, $\left(E_{T}\right)_{a}$, is then given by

$$
\left(E_{T}\right)_{a}=E_{L}+\left(E_{\text {Relax }}\right)_{a},
$$

where the subscript $a$ indicates the energy after the relaxation due to monopole fields takes place, and $\left(E_{\text {Relax }}\right)_{a}$ represents the relaxation energy which consists of the changes in the Madelung, the repulsive, and van der Waals energies, $\Delta E_{M}, \Delta E_{R}$, and $\Delta E_{\mathrm{vdW}}$, and the polarization energy $E_{p}$ (see Refs. 27, 36, and 37).

Furthermore, another relaxation, due to electron-phonon interactions, occurs, and $\mathrm{Ti}^{3+}-\mathrm{Ti}^{3+}$ couples in the $c$ direction are paired. Then the total energy in $\mathrm{Ti}_{2} \mathrm{O}_{3}$ has the form

$$
\left(E_{T}\right)_{b}=E_{L}+\left(E_{\text {Relax }}\right)_{b}+E_{e-\mathrm{ph}},
$$

where the subscript $b$ indicates the energies after the electron-phonon interactions are considered, and the last term, $E_{e \text {-ph }}$, represents the electron-phonon interaction energy. The description on the equilibrium equations involving the electron-phonon interactions was repeated in our previous literature. ${ }^{36,49-53}$ In the end, the following relations were obtained, ${ }^{27}$ and are used to estimate the magnitudes for $\lambda$ and $c$ :

$$
\begin{gathered}
-\frac{\lambda^{2}}{2 c}=\left(E_{T}\right)_{b}-\left(E_{T}\right)_{a}=-\left(E_{T}\right)_{a-b}=E_{D}+E_{e-\mathrm{ph}}, \\
\frac{1}{2} c \sum_{i \neq j} x_{i j}^{2}=\left(E_{\text {Relax }}\right)_{b}-\left(E_{\text {Relax }}\right)_{a}=E_{D},
\end{gathered}
$$

where $x_{i j}$ is the change in the ionic spacing between ions, $i$ and $j$, which is induced by the relaxation-process due to the electron-phonon interactions. The subscript $a-b$ refers to the energy difference between the lattices before and after $\mathrm{Ti}^{3+}-\mathrm{Ti}^{3+}$ couples are paired in the $c$ direction by the electron-phonon interactions. In Eq. (5), ' $E_{D}$ ', represents an increase in the lattice energy due to the local lattice distortion induced by formation of $\mathrm{Ti}^{3+}-\mathrm{Ti}^{3+}$ pairs.

Based upon the Mott-Hubbard regime, the band gap between the upper and lower Hubbard bands, $E_{G}$, is generally defined as ${ }^{36}$

$$
E_{G}=U-\frac{1}{2}\left(B_{1}+B_{2}\right)+\frac{\lambda^{2}}{2 c},
$$

where $U, B_{1}$, and $B_{2}$ are the intersite electron-electron interaction including the spin-spin interaction, the upper Hubbard bandwidth, and the lower Hubbard bandwidth respectively. In the insulating $\mathrm{Ti}_{2} \mathrm{O}_{3}$ phase, the unoccupied $e_{g}^{\pi}$ band belongs to the upper Hubbard band, and the occupied $a_{1 g}$ band is the lower one. Then " $U$ ' quantifies the degree of the contribution of the correlation effects for $d$ electrons, whereas " $\lambda{ }^{2} / 2 c$ " measures that of the electron-phonon interaction effect which is involved only in the calculation relevant to the occupied $a_{1 g}$ band in the insulating phase. Every term in Eq. (6) is calculated by using the DV- $X \alpha$ cluster method, except the last one. One should notice that the calculation in the insulating phase using the cluster method is also based upon the ionic positions of the initial structure 


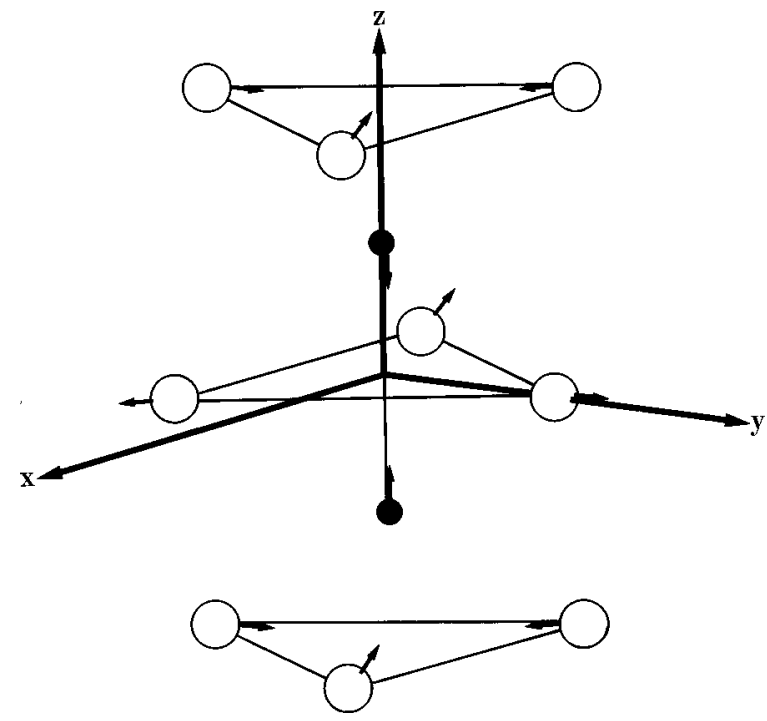

FIG. 2. Geometric structure of the cluster employed in the DV- $X \alpha$ cluster method, i.e., the $\left[\mathrm{Ti}_{2} \mathrm{O}_{9}\right]^{-12}$ cluster which is used in high-temperature metallic phase and low-temperature insulating phase, where open circles represent $\mathrm{O}^{2-}$ ions and closed ones $\mathrm{Ti}^{3+}$. All ions in the metallic phase are arranged in the special positions defined by the atomic parameters $z$ and $x$, while the ionic positions in the initial structure determined by the periodic shell model are employed in the insulating phase. The ionic motions in the lowfrequency $A_{1 g}$ vibration are represented by short arrows.

determined by the shell model. Therefore, the combination of the cluster method and the shell model is the indispensable means for the energy calculation relevant to the transition phenomenon in $\mathrm{Ti}_{2} \mathrm{O}_{3}$.

In order to calculate the electronic structures in both the metallic and insulating $\mathrm{Ti}_{2} \mathrm{O}_{3}$ phases using an ab initio molecular-orbital method, the $\left[\mathrm{Ti}_{2} \mathrm{O}_{9}\right]^{-12}$ cluster is employed, as illustrated in Fig. 2, in which the coordinate $X$ and $Y$ axes perpendicular to the $c$ axis, and the $Z$ axis parallel to the $c$ direction, are indicated. In the metallic phase, all ions were arranged in the special positions defined by the atomic parameters $z$ and $x$. These clusters involve 128 electrons, and the average net charges of titanium and oxygen ions are $+1.56 e$ and $-1.68 e$, respectively. Furthermore, the numerical atomic basis functions, Ti; $1 s \sim 4 p$ and $\mathrm{O} ; 1 s \sim 2 p$, were used to expand the molecular orbitals. These basis functions are the Slater-type orbital basis sets obtained from the short Herman-Skillman programs. ${ }^{72}$

The calculations used the DV-X $\alpha$ calculation program constructed by Satoko ${ }^{39-43}$ for MS-DOS (Microsoft Disk Operation System) personal computers. The DV-X $\alpha$ method, based upon the self-consistent-field Hartree-FockSlater (HFS) one-electron model ${ }^{73}$ and the self-consistentcharge procedure ${ }^{38}$ is one of the most useful techniques for approximately solving the HFS molecular equation. This method is the molecular cluster method, using a local oneelectron effective potential to approximate both the electron correlation effect and the spin correlation effect. Then, the effective Slater $X \alpha$ exchange-correlation potential is given by $-3 \alpha[3 / 8 \pi \rho]^{1 / 3}$, where $\rho$ is the local electron density, and the exchange-scaling parameter $\alpha$ was fixed at 0.7 in the present calculation. In fact, electronic structures in several ionic crystals ${ }^{40-44}$ were investigated by this method. Since
TABLE II. Differences in the Madelung energy $\left(\Delta E_{M}\right)$, repulsive energy $\left(\Delta E_{R}\right)$, and van der Waals energy $\left(\Delta E_{\mathrm{vdW}}\right)$ before and after electron-phonon interactions are considered, and polarization energy $\left(E_{p}\right)$ for the insulating $\mathrm{Ti}_{2} \mathrm{O}_{3}$ phases at 300 and $390 \mathrm{~K}$. These differences are denoted by subscript of $a-b$. The increase in the lattice energy due to formation of $\mathrm{Ti}^{3+}-\mathrm{Ti}^{3+}$ pairs, $E_{D}$, the electron-phonon interaction energy $E_{e \text {-ph }}$, the electron-phonon coupling constant between $\mathrm{Ti}^{3+}$ ions, $\lambda$, and the relevant elastic constant $c$ are also tabulated. Every term is obtained per $\mathrm{Ti}_{2} \mathrm{O}_{3}$ molecule.

\begin{tabular}{ccccc}
\hline \hline $\begin{array}{c}T \\
(\mathrm{~K})\end{array}$ & $\begin{array}{c}\left(\Delta E_{M}\right)_{a-b} \\
(\mathrm{eV})\end{array}$ & $\begin{array}{c}\left(\Delta E_{R}\right)_{a-b} \\
(\mathrm{eV})\end{array}$ & $\begin{array}{c}\left(\Delta E_{\mathrm{vdW}}\right)_{a-b} \\
(\mathrm{eV})\end{array}$ & $\begin{array}{c}\left(E_{p}\right)_{a-b} \\
(\mathrm{eV})\end{array}$ \\
\hline 300 & -0.172 & 0.070 & -0.002 & 0.061 \\
390 & -0.128 & 0.052 & -0.002 & 0.055 \\
$T$ & $E_{D}$ & $E_{e-\mathrm{ph}}$ & $\lambda$ & $\mathrm{c}$ \\
$(\mathrm{K})$ & $(\mathrm{eV})$ & $(\mathrm{eV})$ & $(\mathrm{eV} / \AA)$ & $\left(\mathrm{eV} / \AA^{2}\right)$ \\
\hline 300 & 0.043 & -0.069 & -14.4 & 3988 \\
390 & 0.023 & -0.032 & -10.9 & 6363 \\
\hline \hline
\end{tabular}

Adachi, Tsukada, and Satoko ${ }^{40}$ reported the details of the computational treatment of the DV-X $\alpha$ method, the present calculation has followed.

\section{RESULTS AND DISCUSSION}

\section{A. Energy parameters}

Table II tabulates energy terms per $\mathrm{Ti}_{2} \mathrm{O}_{3}$ molecule calculated by the periodic shell model at 300 and $390 \mathrm{~K}$. The energy units per $\mathrm{Ti}_{2} \mathrm{O}_{3}$ molecule are employed because every energy was evaluated per $\mathrm{Ti}^{3+}-\mathrm{Ti}^{3+}$ pair. As shown in Eq. (4), the difference in the total lattice energy, $\left(E_{T}\right)_{a-b}$, is $\lambda^{2} / 2 c$, and is also obtained by the specific sum of $\left(\Delta E_{M}\right)_{a-b}+\left(\Delta E_{R}\right)_{a-b}+\left(\Delta E_{\mathrm{vdW}}\right)_{a-b}+\left(E_{p}\right)_{a-b}-E_{e-\mathrm{ph}} \quad$ (see Ref. 27). The energy terms in Table II yield $\left(E_{T}\right)_{a-b}$ $=0.026$ and $0.009 \mathrm{eV}$ at 300 and $390 \mathrm{~K}$, respectively. These agree with the values of $\lambda^{2} / 2 c$ which are evaluated by numerical substitutions of $\lambda$ and $c$ in Table II. Since the electrons on $\mathrm{a} \mathrm{Ti}^{3+}$ site are stabilized by the electron-phonon interactions which draw the neighboring $\mathrm{Ti}^{3+}$ ions to the electrons, the spacing of the $\mathrm{Ti}^{3+}-\mathrm{Ti}^{3+}$ pair decreases, i.e., $x_{i j}<0$ for the $\mathrm{Ti}^{3+}-\mathrm{Ti}^{3+}$ pair. Thus the stability of the $\mathrm{Ti}^{3+}-\mathrm{Ti}^{3+}$ pairs requires a negative value for $E_{e \text {-ph }}$, accordingly, $\lambda$ must be negative because $E_{e-\mathrm{ph}}=-\lambda \Sigma x_{i j}$ (see Ref. 27). The energy values in Table II are surely subject to this requirement. Consequently the periodic shell model provides direct evidence that $\mathrm{Ti}^{3+}-\mathrm{Ti}^{3+}$ pairs are stabilized and form singlet excitons in the insulating $\mathrm{Ti}_{2} \mathrm{O}_{3}$ phase, that is, this phase is stable. The magnitudes for $\lambda$ in $\mathrm{Ti}_{2} \mathrm{O}_{3}$, i.e., $-14.4 \mathrm{eV} / \AA$ at $300 \mathrm{~K}$ and $-10.9 \mathrm{eV} / \AA$ at $390 \mathrm{~K}$, are considerably smaller in comparison with that in $\mathrm{VO}_{2}$, i.e., $-35.2 \mathrm{eV} / \AA$. It is also noteworthy that the magnitude for $-\lambda$ decreases with increasing temperature.

In order to investigate the transformation of the electronic structure due to the transition in $\mathrm{Ti}_{2} \mathrm{O}_{3}$, the DV- $X \alpha$ cluster calculation was carried out. Figure 2 shows the structure of the $\left[\mathrm{Ti}_{2} \mathrm{O}_{9}\right]^{-12}$ cluster used in the calculation. In the MottHubbard regime, the energy gap between the upper and lower Hubbard bands is generally formulated by Eq. (6). 
TABLE III. Upper Hubbard bandwidth $B_{1}$, lower Hubbard bandwidth $B_{2}$, intersite $d$ - $d$ interaction energy including the spinspin interaction, $U$, the magnitude for $\lambda^{2} / 2 c$ which comes from the Anderson's attractive potential, and band gap $E_{G}$, at several temperatures from 300 to $868 \mathrm{~K}$. Every term is in the units of eV.

\begin{tabular}{lccccc}
\hline \hline$T(\mathrm{~K})$ & $B_{1}$ & $B_{2}$ & $U$ & $\lambda^{2} / 2 c$ & $E_{G}$ \\
\hline 300 & 1.820 & 1.002 & 1.483 & 0.026 & 0.098 \\
390 & 1.810 & 1.000 & 1.449 & 0.009 & 0.053 \\
450 & 1.728 & 0.980 & 1.354 & & \\
490 & 1.656 & 0.974 & 1.315 & & \\
565 & 1.586 & 0.934 & 1.260 & & \\
621 & 1.543 & 0.917 & 1.230 & & \\
713 & 1.528 & 0.912 & 1.220 & & \\
868 & 1.466 & 0.879 & 1.173 & & \\
\hline \hline
\end{tabular}

Each term on the right-hand side of this equation has been calculated using the cluster method and the periodic shell model, and then these terms yield the energy gap $E_{G}$. That is, opening the gap between the occupied $a_{1 g}$ band and the unoccupied $e_{g}^{\pi}$ band in the insulating phase is obtained by taking account of the electron correlation effect and the spin correlation effect quantified by $U$, and the electron-phonon interaction effect represented by $\lambda^{2} / 2 c$. Table III tabulates the magnitudes for $B_{1}, B_{2}, U, \lambda^{2} / 2 c$, and $E_{G}$ in the insulating phases, together with those for $B_{1}, B_{2}$, and $U$ in the metallic phases.

\section{B. Electronic structures}

Figures 3(a) and 3(b) illustrate the resultant electronic structures in the metallic phase at $490 \mathrm{~K}$ and the insulating phase at $300 \mathrm{~K}$, which are constructed using the energy parameters in Tables II and III. The most important point in the present results is that the upper and lower Hubbard bands overlap at the Fermi level in the high-temperature metallic phase, as shown in Fig. 3(a). In the low-temperature insulating phase, as shown in Fig. 3(b), there is obviously an energy gap between the occupied $a_{1 g}$ band and the unoccupied $e_{g}^{\pi}$ band which overlaps partially the $a_{1 g}^{*}$ band, where the asterisk indicates an antibonding orbital. Therefore this transformation of the band structure provides theoretical direct evidence of the transition phenomenon in $\mathrm{Ti}_{2} \mathrm{O}_{3}$. Moreover, the estimated magnitude of $E_{G}=0.098 \mathrm{eV}$ agrees well with the experimental result, i.e., $E_{G} \simeq 0.1 \mathrm{eV}$ at $300 \mathrm{~K}^{2-6}$ In $\mathrm{VO}_{2}$, the electron-phonon interaction effect is an indispensable contribution to the MI transition because the band gap never opens if this term is not included in the calculations. As shown in Table III, $\lambda^{2} / 2 c=0.026 \mathrm{eV}$ at $300 \mathrm{~K}$ and $0.009 \mathrm{eV}$ at $390 \mathrm{~K}$ which are considerably small in values compared with that in $\mathrm{VO}_{2}$, i.e., $0.44 \mathrm{eV}$. This fact reconfirms the weak electron-phonon interaction effect in $\mathrm{Ti}_{2} \mathrm{O}_{3}$, as suggested in the past. ${ }^{22}$ In fact, without the assistance of the electronphonon interactions, the $d$ - $d$ electron correlation effect and the spin correlation effect can open the band gap in the insulating phase, yielding $E_{G}=0.072 \mathrm{eV}$ at $300 \mathrm{~K}$, which is, however, somewhat lower than the experimental value. Furthermore, Table III indicates that the electron-phonon interaction effect decays rapidly as temperature rises to the starting point of the transition. This result implies that the

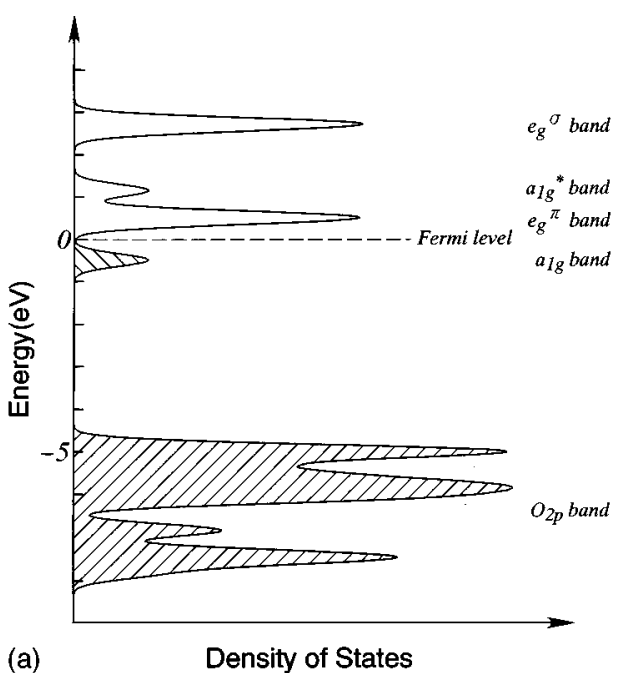

(a)

Density of States

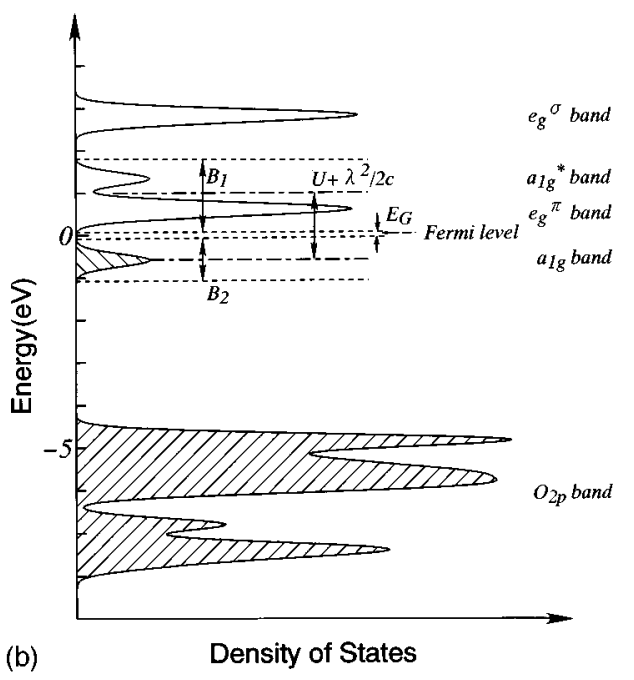

FIG. 3. Electronic structures of $\mathrm{Ti}_{2} \mathrm{O}_{3}$ near the Fermi level. (a) High-temperature metallic phase at $490 \mathrm{~K}$. (b) Low-temperature insulating phase at $300 \mathrm{~K}$. The electronic structure of the metal phase was obtained by the DV-X $\alpha$ cluster method, while that in the insulating phase by the combination of the DV-X $\alpha$ cluster method and the periodic shell model. The occupied bands are shaded. The Fermi energy is taken as the standard level.

electron-phonon interaction effect in $\mathrm{Ti}_{2} \mathrm{O}_{3}$ is less significant in the origin of the transition phenomenon if compared with $\mathrm{VO}_{2}$, but never neglected. This is consistent with the prediction of Ashekenzai and Chuchem. ${ }^{22}$

The theoretical treatment in the present paper explains rather well the characteristic transformation of the electronic structure causing the broad crossover between the insulating and metallic states. The features in Figs. 3(a) and 3(b) are in good agreement with the proposal of Goodenough and coworkers, i.e., the band-crossing model in $\mathrm{Ti}_{2} \mathrm{O}_{3} \cdot{ }^{19-21}$ The crystalline field splits the fivefold-degenerate $d$ orbitals into a pair of $e_{g}^{\sigma}$ orbitals directed toward nearest-neighbor $\mathrm{O}^{2-}$ ions, a pair of $e_{g}^{\pi}$ orbitals involved in the bonding between $\mathrm{Ti}^{3+}$ ions in the basal plane perpendicular to the $c$ axis, and an $a_{1 g}$ orbital directed between the $\mathrm{Ti}^{3+}-\mathrm{Ti}^{3+}$ pairs lying along the $c$ axis. The $a_{1 g}$ band can contain up to two electrons per " $\mathrm{Ti}_{2} \mathrm{O}_{3}$ "' molecule and the $e_{g}^{\pi}$ band eight electrons. 


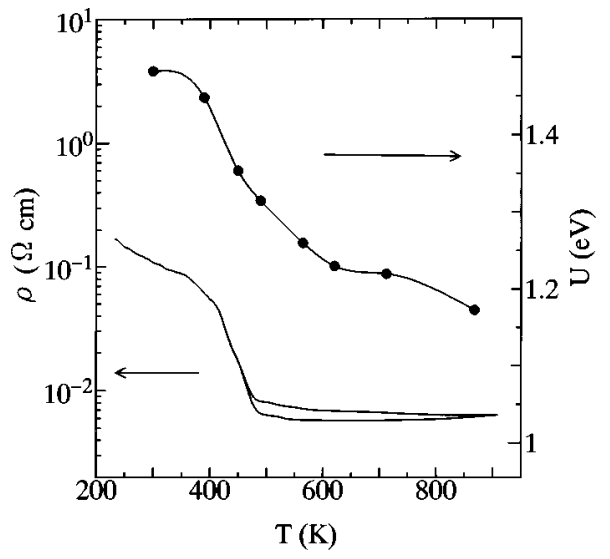

FIG. 4. Resistivities and calculated $U$ values as a function of temperature for $\mathrm{Ti}_{2} \mathrm{O}_{3}$.

The remaining $a_{1 g}^{*}$ and $e_{g}^{\sigma}$ bands can together hold ten electrons per molecular unit. In the metallic phase, $\mathrm{Ti}_{2} \mathrm{O}_{3}$ has a rather large $c_{H} / a_{H}$ ratio, so that a thermal excitation of electrons can be enhanced from the $a_{1 g}$ band to the $e_{g}^{\pi}$ band, ${ }^{19}$ as shown in Fig. 3(a). In the insulating phase, $\mathrm{Ti}_{2} \mathrm{O}_{3}$ has a rather small $c_{H} / a_{H}$ ratio, and forms singlet excitons, so that the relative position of the occupied $a_{1 g}$ band decreases more in energy from the unoccupied $e_{g}^{\pi}$, band which is between the $a_{1 g}$ and $a_{1 g}^{*}$ bands, as illustrated in Fig. 3(b). Calculations were carried out at several temperatures where the lattice parameters are available experimentally.

\section{Comparison with other studies}

The transition behavior in $\mathrm{Ti}_{2} \mathrm{O}_{3}$ is definitely different from that of $\mathrm{VO}_{2} \cdot \mathrm{VO}_{2}$ exhibits a clear cut MI transition, while a broad crossover transition takes place in $\mathrm{Ti}_{2} \mathrm{O}_{3}$. Castellani, Feinberg, and Ranninger ${ }^{26}$ calculated the gap in the density of states and the nearest-neighbor spin correlation function for the $\mathrm{Ti}^{3+}-\mathrm{Ti}^{3+}$ pairs along the $c$ axis as a function of the interpair hopping integral in $\mathrm{Ti}_{2} \mathrm{O}_{3}$. In their theoretical treatment, the electron-phonon interaction assessed by the relative relationship between the intrapair hopping integral and the interpair hopping integral decays with decreasing the magnitudes for the gap and the spin correlation function. Particularly, their calculation indicates the possibility of a broad crossover in the transition due to the antiferromagnetic-diamagnetic phase transition which relates closely to the spin correlation effect.

The general feature obtained in the present study agrees with their result. ${ }^{26}$ Figure 4 demonstrates the temperature dependence of resistivities in $\mathrm{Ti}_{2} \mathrm{O}_{3}$ obtained by Morin, ${ }^{1}$ together with the calculated $U$ values which include both the electron correlation effect and the spin correlation effect. In the insulating phase at $300 \mathrm{~K}, U$ is $1.483 \mathrm{eV}$, but it reduces to $1.315 \mathrm{eV}$ in the metallic phase at $490 \mathrm{~K}$, and decreases more to $1.173 \mathrm{eV}$ at $868 \mathrm{~K}$ with increasing temperature. This means that $a_{1 g}$ and $e_{g}^{\pi}$ bands cross over one another more deeply in the metallic phase as temperature increases. As indicated in our previous paper, ${ }^{27}$ the clear cut MI transition in $\mathrm{VO}_{2}$ is mainly due to the lattice transformation, to which the electron-phonon interaction contributes very significantly, while the broad crossover transition in $\mathrm{Ti}_{2} \mathrm{O}_{3}$ does not involve a lattice transformation, in which the electronphonon interaction does not play such an important role (see Table III). In the transition of $\mathrm{Ti}_{2} \mathrm{O}_{3}$, however, the decrease in the correlation effects with increasing temperature plays an important role because of the similar temperature dependence of $U$ values to that of resistivities as shown in Fig. 4. Therefore, present results indicate that the electron correlation effect and the spin correlation effect are mainly responsible for the characteristic transition in $\mathrm{Ti}_{2} \mathrm{O}_{3}$. To our knowledge, there are no reports in the literature which provide direct theoretical evidence of this transition.

The relative positions of the density of states of $a_{1 g}$ and $\mathrm{O}$ $2 p$ bands in the metallic and insulating phase of $\mathrm{Ti}_{2} \mathrm{O}_{3}$ calculated in the present paper are competitive with those obtained by ARUPS studies. ${ }^{17,18}$ Both these experiments and our calculation yield 1.0-1.5 eV for the bandwidth of the $a_{1 \mathrm{~g}}$ band and the upper edge of the $\mathrm{O} 2 p$ band 3.5-4.5 eV below the Fermi level.

Raman spectrum studies ${ }^{10-13}$ observed seven Ramanactive phonon modes allowed in the $D_{3 d}^{6}$ space-group symmetry of the corundum structure for $\mathrm{Ti}_{2} \mathrm{O}_{3}$. Two of these seven modes belong to the totally symmetric modes of $A_{1 \mathrm{~g}}$ symmetry, and the remaining five modes belong to the doubly degenerate modes of $E_{g}$ symmetry. ${ }^{11}$ Recently, femtosecond time-resolved pump-probe reflection experiments were applied to a study of the transition behavior in $\mathrm{Ti}_{2} \mathrm{O}_{3}$, and detected only a low-frequency $A_{1 g}$ mode at room temperature. ${ }^{74-76}$ In fact, Zeiger and co-workers ${ }^{74-76}$ proposed the displacive excitation of coherent phonons mechanism, and concluded that the energy gap was modulated according to the induced coherent lattice vibration associated with the low-frequency $A_{1 g}$ mode. The $A_{1 g}$ modes are breathing modes with no change in lattice symmetry. The motions of titanium and oxygen ions in the low-frequency $A_{1 g}$ mode are shown in Fig. 2. Goodenough and co-workers ${ }^{19-21}$ suggested that the low-frequency $A_{1 g}$ mode enhances deformation of the polarizable $3 d$-electron cloud with increasing $c_{H} / a_{H}$ ratio due to a screening effect of $\mathrm{O}^{2-}$ ions. As $3 d$ electrons are excited from the occupied $a_{1 g}$ band to the unoccupied $e_{g}^{\pi}$ band with increasing temperature, the polarizability of this cloud greatly increases. Finally, a strong resonant enhancement of the intensity of the $E_{g}$ mode is observed, ${ }^{21}$ and the energy gap is collapsed, as shown in Fig. 3(a). The present results are not inconsistent with the Raman spectrum studies.

Zeiger suggested that the collapse of the energy gap was impeded by the thermal excitation of the singlet excitons accompanied by local polaronlike distortion. ${ }^{25}$ This implies that the insulating $\mathrm{Ti}_{2} \mathrm{O}_{3}$ can be treated as a stable phase through local lattice distortion induced by formation of $\mathrm{Ti}^{3+}-\mathrm{Ti}^{3+}$ pairs along the $c$ axis due to the electron-phonon interaction. The present calculation also indicates that this interaction is surely one of the indispensable effects which stabilize the insulating phase. In order to estimate the numerical magnitude of the electron-phonon interaction energy, we employed a technique similar to that which succeeded in accounting for the clear cut MI transition in $\mathrm{VO}_{2},{ }^{27}$ although Castellani, Feinberg, and Ranninger ${ }^{26}$ used another sophisticated method. In the present study, the electron correlation and spin correlation effects in the formation of diamagnetic 
units on two adjacent $\mathrm{Ti}^{3+}$ ions in the $c$ direction are found to participate directly in the driving force for the characteristic broad crossover transition in $\mathrm{Ti}_{2} \mathrm{O}_{3}$, although there is some contribution from the electron-phonon interaction. The results obtained here explain clearly the band-crossing model proposed by Goodenough and co-workers, ${ }^{19-21}$ and the collapse mechanism of the band gap causing the low-frequency $A_{1 g}$ mode suggested by Zeiger and co-workers. ${ }^{74-76}$ The cluster calculation is then one of the most reliable means to account for the transition phenomenon in $\mathrm{Ti}_{2} \mathrm{O}_{3}$ if combined with the periodic shell model.

\section{ACKNOWLEDGMENTS}

The authors are very grateful to M. Tsuji and N. Nakamura for useful discussion and assistance in this project. This project was supported by a Grant-in-Aid for Science Research (No. 08650812) from the Ministry of Education, Science and Culture, Japan.
*Author to whom correspondence should be addressed.

${ }^{1}$ F. J. Morin, Phys. Rev. Lett. 3, 34 (1959).

${ }^{2}$ J. M. Honig and T. B. Reed, Phys. Rev. 174, 1020 (1968).

${ }^{3}$ T. C. Chi and R. J. Sladek, Phys. Rev. B 7, 5080 (1973).

${ }^{4}$ H. L. C. Barros, T. C. Chi, G. V. Chandrashekhar, J. M. Honig, and R. J. Sladek, Phys. Rev. B 7, 5147 (1973).

${ }^{5}$ S. H. Shin, G. V. Chandrashekhar, R. E. Loehman, and J. M. Honig, Phys. Rev. B 8, 1364 (1973).

${ }^{6}$ G. Luckovsky, J. W. Allen, and P. Allen, Inst. Phys. Conf. Ser. 43, 465 (1979)

${ }^{7}$ J. Yahia and H. P. R. Frederikse, Phys. Rev. 123, 1257 (1961).

${ }^{8}$ T. B. Reed, R. E. Fahey, and J. M. Honig, Mater. Res. Bull. 2, 561 (1967).

${ }^{9}$ P. M. Raccah and A. Mooradian, Bull. Am. Phys. Soc. 15, 327 (1970).

${ }^{10}$ A. Mooradian and P. M. Raccah, Phys. Rev. B 3, 4253 (1971).

${ }^{11}$ S. H. Shin, R. L. Aggarwal, B. Lax, and J. M. Honig, Phys. Rev. B 9, 583 (1974).

${ }^{12}$ S. H. Shin, F. H. Pollak, T. Halpern, and P. M. Raccah, Solid State Commun. 16, 687 (1975).

${ }^{13}$ S. H. Shin, F. H. Pollak, T. Halpern, and P. M. Raccah, J. Solid State Chem. 12, 407 (1975).

${ }^{14}$ J. M. Honig and L. L. Van Zandt, Annu. Rev. Mater. Sci. 5, 225 (1975).

${ }^{15}$ G. Lucovsky, R. J. Sladek, and J. W. Allen, Phys. Rev. B 16, 5452 (1977).

${ }^{16}$ J. M. McKay, M. H. Mohamed, and V. E. Henrich, Phys. Rev. B 35, 4304 (1987).

${ }^{17}$ K. E. Smith and V. E. Henrich, Phys. Rev. B 38, 5965 (1988).

${ }^{18}$ K. E. Smith and V. E. Henrich, Phys. Rev. B 38, 9571 (1988).

${ }^{19}$ J. B. Goodenough, Phys. Rev. 117, 1442 (1960).

${ }^{20}$ L. L. Van Zandt, J. M. Honig, and J. B. Goodenough, J. Appl. Phys. 39, 594 (1968).

${ }^{21}$ J. B. Goodenough, Prog. Solid State Chem. 5, 145 (1972).

${ }^{22}$ J. Ashkenazi and T. Chuchem, Philos. Mag. 32, 763 (1975).

${ }^{23}$ J. Ashkenazi and M. Weger, J. Phys. (Paris), Colloq. 37, C4-189 (1976)

${ }^{24}$ H. J. Zeiger, T. A. Kaplan, and P. M. Raccah, Phys. Rev. Lett. 26, 1328 (1971).

${ }^{25}$ H. J. Zeiger, Phys. Rev. B 11, 5132 (1975).

${ }^{26}$ C. Castellani, D. Feinberg, and J. Ranninger, J. Phys. C 12, 1541 (1979).

${ }^{27}$ H. Nakatsugawa and E. Iguchi, Phys. Rev. B 55, 2157 (1997).

${ }^{28}$ C. E. Rice and W. R. Robinson, Mater. Res. Bull. 11, 1355 (1976).

${ }^{29}$ C. E. Rice and W. R. Robinson, Acta Crystallogr., Sect. B: 33, 1342 (1977).

${ }^{30}$ R. E. Newnham and Y. M. de Haan, Z. Kristallogr. 117, 235 (1962).

${ }^{31}$ S. C. Abrahams, Phys. Rev. 130, 2230 (1963).
${ }^{32}$ B. K. Chakraverty and C. S. Schlenker, J. Phys. (Paris), Colloq. 37, C4-353 (1976).

${ }^{33}$ P. W. Anderson, Phys. Rev. Lett. 34, 953 (1975).

${ }^{34} \mathrm{P}$. W. Anderson (private communication).

${ }^{35}$ C. Kittel, Introduction to Solid State Physics, 4th ed. (Wiley, New York, 1971), p. 391.

${ }^{36}$ E. Iguchi and H. Nakatsugawa, Phys. Rev. B 51, 10956 (1995).

${ }^{37}$ G. J. Dienes, D. O. Welch, C. R. Fischer, R. D. Hatcher, O. Lazareth, and M. Samberg, Phys. Rev. B 11, 3060 (1975).

${ }^{38}$ A. Rosën, D. E. Ellis, H. Adachi, and F. W. Averill, J. Chem. Phys. 65, 3629 (1976).

${ }^{39}$ A. Zunger and A. J. Freeman, Phys. Rev. B 15, 4716 (1977).

${ }^{40}$ H. Adachi, M. Tsukada, and C. Satoko, J. Phys. Soc. Jpn. 45, 875 (1978).

${ }^{41}$ C. Satoko, M. Tsukada, and H. Adachi, J. Phys. Soc. Jpn. 45, 1333 (1978).

${ }^{42}$ M. Tsukada, C. Satoko, and H. Adachi, J. Phys. Soc. Jpn. 47, 1610 (1979).

${ }^{43}$ M. Tsukada, C. Satoko, and H. Adachi, J. Phys. Soc. Jpn. 48, 200 (1980).

${ }^{44}$ K. Goto, F. Munakata, M. Yamanaka, and H. Adachi, J. Solid State Chem. 119, 76 (1995).

${ }^{45}$ H. Sawatari, E. Iguchi, and R. J. D. Tilley, J. Phys. Chem. Solids 43, 1147 (1982).

${ }^{46}$ K. Aizawa, E. Iguchi, and R. J. D. Tilley, Proc. R. Soc. London, Ser. A 394, 299 (1984).

${ }^{47}$ E. Iguchi and F. Matsushima, J. Phys. Chem. Solids 47, 45 (1986).

${ }^{48}$ E. Iguchi and T. Yamamoto, J. Phys. Chem. Solids 49, 205 (1988).

${ }^{49}$ E. Iguchi, A. Tamenori, and N. Kubota, Phys. Rev. B 45, 697 (1992).

${ }^{50}$ E. Iguchi and K. Akashi, J. Phys. Soc. Jpn. 61, 3385 (1992).

${ }^{51}$ E. Iguchi, K. J. Lee, and A. Iguchi, J. Phys. Soc. Jpn. 62, 1135 (1993).

${ }^{52}$ E. Iguchi and H. Miyagi, J. Phys. Chem. Solids 54, 403 (1993).

${ }^{53}$ E. Iguchi, T. Hashimoto, and S. Yokoyama, J. Phys. Soc. Jpn. 65, 221 (1996).

${ }^{54}$ P. P. Ewald, Ann. Phys. (N.Y.) 64, 253 (1921).

${ }^{55}$ W. Van Gool and A. G. Piken, J. Mater. Sci. 4, 95 (1969).

${ }^{56}$ P. T. Wedepohl, Proc. Phys. Soc. London 92, 79 (1967).

${ }^{57}$ P. T. Wedepohl, J. Phys. C 10, 1855 (1977).

${ }^{58}$ P. T. Wedepohl, J. Phys. C 10, 1865 (1977).

${ }^{59}$ R. E. Watson, Phys. Rev. 111, 1108 (1958).

${ }^{60}$ A. R. Ruffa, Phys. Rev. 130, 1412 (1963).

${ }^{61}$ A. R. Ruffa, Phys. Rev. 133, A1418 (1964).

${ }^{62}$ R. A. Parker, Phys. Rev. 124, 1713 (1961).

${ }^{63}$ E. Iguchi, K. Ohtake, T. Yamamoto, and H. Nishikawa,J. Nucl. Mater. 169, 55 (1989). 
${ }^{64}$ E. Iguchi and Y. Yonezawa, J. Phys. Chem. Solids 51, 313 (1990).

${ }^{65}$ E. Iguchi, H. Sugimoto, A. Tamenori, and H. Miyagi, J. Solid State Chem. 91, 286 (1991).

${ }^{66}$ E. Iguchi, H. Nishikawa, A. Tamenori, T. Manako, and H. Igarashi, Physica C 179, 393 (1991).

${ }^{67}$ L. Pauling, Proc. R. Soc. London, Ser. A 114, 181 (1927).

${ }^{68}$ B. G. Dick and A. W. Overhauser, Phys. Rev. 112, 90 (1958).

${ }^{69}$ J. S. Shanker, Indian J. Pure Appl. Phys. 11, 381 (1973).

${ }^{70}$ J. S. Shanker and V. P. Gupta, J. Phys. Chem. Solids 41, 141 (1980).

${ }^{71}$ R. D. Shannon and C. T. Prewitt, Acta Crystallogr., Sect. B: Struct. Sci. 26, 1046 (1970).
${ }^{72}$ F. Herman and S. Skillman, Atomic Structure Calculations (Prentice-Hall, Englewood Cliffs, NJ, 1963), p. 1.

${ }^{73}$ J. C. Slater, Quantum Theory of Molecules and Solids (McGrawHill, New York, 1974), Vol. 4.

${ }^{74}$ H. J. Zeiger, J. Vidal, T. K. Cheng, E. P. Ippen, G. Dresselhaus, and M. S. Dresselhaus, Phys. Rev. B 45, 768 (1992).

${ }^{75}$ T. K. Cheng, L. H. Acioli, J. Vidal, H. J. Zeiger, G. Dresselhaus, M. S. Dresselhaus, and E. P. Ippen, Appl. Phys. Lett. 62, 1901 (1993).

${ }^{76}$ H. J. Zeiger, T. K. Cheng, E. P. Ippen, J. Vidal, G. Dresselhaus, and M. S. Dresselhaus, Phys. Rev. B 54, 105 (1996). 